\title{
Cheap talk with multiple strategically interacting audiences: An experimental study
}

Citation for published version (APA):

Li, X., \& Peeters, R. J. A. P. (2013). Cheap talk with multiple strategically interacting audiences: An experimental study. Maastricht University, Graduate School of Business and Economics. GSBE Research Memoranda No. 035 https://doi.org/10.26481/umagsb.2013035

Document status and date:

Published: 01/01/2013

DOI:

10.26481/umagsb.2013035

Document Version:

Publisher's PDF, also known as Version of record

\section{Please check the document version of this publication:}

- A submitted manuscript is the version of the article upon submission and before peer-review. There can be important differences between the submitted version and the official published version of record.

People interested in the research are advised to contact the author for the final version of the publication, or visit the DOI to the publisher's website.

- The final author version and the galley proof are versions of the publication after peer review.

- The final published version features the final layout of the paper including the volume, issue and page numbers.

Link to publication

\footnotetext{
General rights rights.

- You may freely distribute the URL identifying the publication in the public portal. please follow below link for the End User Agreement:

www.umlib.nl/taverne-license

Take down policy

If you believe that this document breaches copyright please contact us at:

repository@maastrichtuniversity.nl

providing details and we will investigate your claim.
}

Copyright and moral rights for the publications made accessible in the public portal are retained by the authors and/or other copyright owners and it is a condition of accessing publications that users recognise and abide by the legal requirements associated with these

- Users may download and print one copy of any publication from the public portal for the purpose of private study or research.

- You may not further distribute the material or use it for any profit-making activity or commercial gain

If the publication is distributed under the terms of Article $25 \mathrm{fa}$ of the Dutch Copyright Act, indicated by the "Taverne" license above, 


\section{Maastricht University}

Xinyu Li, Ronald Peeters

Cheap talk with multiple strategically interacting audiences: An experimental study

$\mathrm{RM} / 13 / 035$

\section{GSBE}

Maastricht University School of Business and Economics

Graduate School of Business and Economics

P.O Box 616

NL- 6200 MD Maastricht

The Netherlands 


\title{
Cheap talk with multiple strategically interacting audiences: An experimental study*
}

\author{
Xinyu $\mathrm{Li}^{\dagger} \quad$ Ronald Peeters ${ }^{\ddagger}$
}

June 26, 2013

\begin{abstract}
We consider a cheap-talk setting that mimics the situation where an incumbent firm (the sender) is endowed with incentives to understate the true size of the market demand to two potential entrants (the receivers). Although our experimental data reveals that senders' messages convey truthful information and this is picked up by the receivers, this overcommunication (relative to standard theoretical prediction) does not enhance efficient entry levels (and payoffs) to beyond what can be achieved without any communication. The reason is that receivers fail to optimally translate the information received in their entry decision, possibly due to overcautiousness.
\end{abstract}

JEL Classification: C72, C92, D82, D83.

Keywords: strategic information transmission, cheap talk, experiment.

*We like to thank Roberto Hernán González, Marc Vorsatz and the audiences in Maastricht (MLSE), Madrid (IMEBE 2013), Florence (BEELab 2013) and Zürich (ESA 2013) for their useful suggestions and feedback. The second author thanks the Netherlands Organisation for Scientific Research (NWO) for financial support.

${ }^{\dagger}$ Department of Economics, Maastricht University. E-mail: x.li@maastrichtuniversity.nl

${ }^{\ddagger}$ Department of Economics, Maastricht University. E-mail: r.peeters@maastrichtuniversity.nl 


\section{Introduction}

In various economic environments of strategic information transmission, the message sent by an informed agent may simultaneously affect actions of multiple, possibly strategically interacting, uninformed agents. One interesting two-audience communication problem, that is mentioned in Farrell and Gibbons (1989), is that of a firm's choice among accounting conventions. In an inflationary era, an inventory-carrying firm may report low profits (by calculating costs according to the LIFO convention) or high ones (adopting FIFO). While for reasons of taxation the former may be interesting, for stock market reasons it is definitely more interesting to use the latter option. Farrell and Gibbons show that the presence of multiple audiences may induce public messages to become informative where messages would not carry any information in any isolated bilateral interaction, but can also complicate information revelation where this would happen in all isolated bilateral communications. All depends on the level of agreement of the alignments of the preferences with the different audiences.

In a controlled laboratory experiment, Battaglini and Makarov (2012) examine the effect of adding one audience on the information transmission process and find that it alters the communication in a way that is consistent with the theoretical predictions by Farrell and Gibbons. Like Battaglini and Makarov, we examine strategic information transmission with multiple audiences in a controlled laboratory experiment. In contrast, where they restrict attention to the situation where the payoffs of the receivers are independent of each other's actions (in spirit of Farrell and Gibbons), we assume strategic interdependence between the two receivers.

The cheap talk setting (treatment CT) that we consider in our experiment resembles the situation in which an incumbent firm can signal market conditions to two possible entrants via one public message. The three possible market conditions refer to the demand in the market, which is either small, medium or large. In this situation, independent of the state, the incumbent has a preference to deter entry. The incumbent can achieve this by having the potential entrants believing that the demand is small, in which case it is a dominant action for them not to enter. As a result, only babbling equilibria exist (cf. Sobel, 2009). Where potential entrants should not enter in case of a small market, there are benefits for both when entering in case the market is large; in case of a medium sized market, there is room for only one potential entrant to enter with positive benefit. So, regarding the alignment of preferences between the incumbent and the potential entrants, there is perfect alignment in a small market and they are perfect antagonists in a large market; in the medium sized market it is somewhere in between.

Despite there only being babbling equilibria, from an ex ante perspective, all parties 
involved would prefer full revelation to any babbling equilibrium. Thus, there is a mutual benefit to ameliorate the failure to communicate. In order to assess whether cheap talk yields more efficient entry levels and higher payoffs, we implement two baseline treatments: one in which full information is given to the potential entrants (treatment FI) and one in which the communication channel is disabled (treatment NI). If the overcommunication (relative to sequential equilibrium assuming rational and egoistic agents) that is found in one-sender/onereceiver experiments (cf. Dickhaut et al., 1995; Cai and Wang, 2006; Sánchez-Pagés and Vorsatz, 2007; and Wang et al., 2010), is also found in our one-sender/two-receivers setting (as in Battaglini and Makarov, 2012), there is scope for cheap talk to generate additional surplus relative to the situation without information revelation.

Our experimental findings are that cheap talk does not generate any efficiency of entry and payoffs beyond the level that is obtained without information transmission. On average, senders' (incumbents) messages convey truthful information. In case senders send deceptive messages, most of the time it concerns strategic deception (understating the state), but there is a robust substantial amount of obscure deceptions (overstating the state). The receivers' (potential entrants) empirical probability to enter is increasing in the size of the market as it is claimed to be by the sender. Thus, despite cheap talk not being able to enhance overall performance, messages contain relevant information and this is picked up by the receivers. So, while we find overcommunication (relative to standard theoretical prediction) in the laboratory, compared to the situation without information transmission, cheap talk doesn't work to alleviate the asymmetric information problem. The reason is that receivers fail to optimally translate the information received in their entry decision, possibly due to overcautiousness.

In the next section we present the experimental design and hypotheses. Section 3 deals with the data analysis and we search for further evidences that may explain our main results in Section 4.

\section{Experiment}

In this section we present, subsequently, the central cheap talk setting, the treatments we implemented in our experiment and the corresponding hypotheses (based on standard theoretical prediction), and details on the precise design and procedures followed.

\section{$2.1 \quad$ Setting}

We consider a signaling game with one sender and two receivers. The game may be in one of three possible states of the world; each state being equally likely a priori. The sender is privately informed on the actual state and has the ability to inform the receivers on the actual 
state via one public message notifying both receivers about the state being drawn. The only information the receivers have about the true state is the message sent to them and they have to choose independently out of two alternative actions: In or Out. The action choices of both receivers together with the true state of the world, determines the payoffs of the three players. The resulting payoffs are displayed in Table 1. In each cell, corresponding to the choices of the row receiver and the column receiver, the first entry presents the sender's payoff, the second the row receiver's, and the third the column receiver's payoff. Notice that the payoffs do not depend on the message being sent; so, talk is cheap.

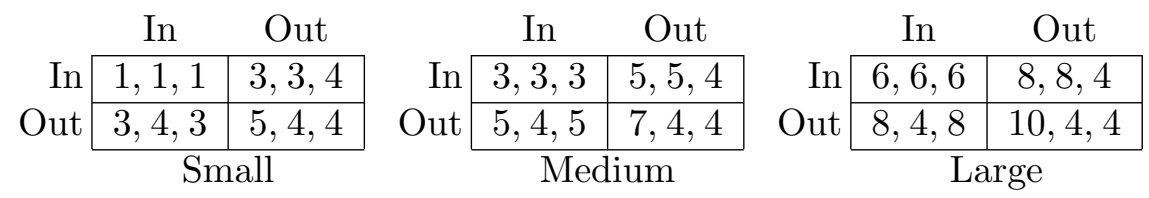

Table 1: The signaling game with two strategically interacting receivers.

This setting is a natural analogue of an entry game with one incumbent and two potential entrants and where the states of the world correspond to possible market sizes: small, medium or large. The potential entrants have no experience in the market that they consider entering, apart from the three states being equally likely, and nevertheless have to decide whether to enter or to stay out. By staying out, the potential entrant can get a sure reservation profit of 4. When entering, the resulting profit may be smaller or larger, depending on the true state and on the entry decision of the other potential entrant. In general, everything else equal, the potential profit of an actual entrant is larger if the market is larger in size and larger with less competitors in the market. This does not hold only for the potential entrants, but is also a structural characteristic of the incumbents profit. Therefore, the incumbent has an incentive to deceive the potential entrants by understating the state of the world when giving the opportunity to provide information on the market condition to them. Of course, the potential entrants are aware of these possibility of the preferences being misaligned in better market circumstances.

\subsection{Treatments and hypotheses}

Our primary treatment - which we dub the cheap talk (CT) treatment - is the setting described above. Apart from understanding how information is strategically transmitted in such a setting involving multiple strategically interacting receivers, we are interested whether cheap talk can ameliorate part of the information disparity between the sender on the one hand and the receivers on the other hand. In order to make such a comparison, we have two baseline treatments: the full information (FI) treatment and the no information (NI) 
treatment. The latter baseline positions receivers in the worst situation where no information about the state is given; the former baseline places them in the ideal situation where they have complete information on the state.

In case the receivers are fully informed about the actual state of the worlds (treatment FI), both will stay out in the small state and will enter in the large state; in the medium state there are three equilibria: two where precisely one of the receivers enters and one in which each of them enters with fifty percent probability.

The case where the receivers have no information at all (treatment NI), possesses three equilibria: two pure asymmetric equilibria in which one of the receivers enters while the other stays out and one symmetric mixed equilibrium in which each of the receivers enters with two-thirds probability. Comparing these payoffs with those in the FI treatment learns that all players involved prefer, from an ex ante perspective, the FI treatment over the NI treatment.

As in the cheap talk setting (treatment CT), the sender always attempts the receivers to believe that the true state is the small one, all messages loose their credibility. As a result, there are no separating equilibria. All "babbling equilibria" render the same outcomes as predicted for the NI treatment.

We can compare the performance of the CT treatment with the two baselines on the basis of two efficiency criteria: the first regards the frequency of efficient entries (that is, entry until excess profits are eroded), the second regards the payoffs generated. Table 2 summarizes the expected frequency of efficient entry levels in each of the states and the ex ante expected payoffs to the sender and receiver for each of the three treatments, as predicted by the symmetric equilibrium in the respective treatment.

\begin{tabular}{lccccccc}
\hline \multirow{2}{*}{ Treatment } & \multicolumn{3}{c}{ Frequency of efficient entry levels } & & \multicolumn{2}{c}{ Expected payoff } \\
\cline { 2 - 4 } & Small & Medium & Large & Average & & Sender & Receiver \\
\hline Full info (FI) & 1.00 & 0.50 & 1.00 & 0.83 & & 5.33 & 4.67 \\
No info (NI) & 0.11 & 0.44 & 0.44 & 0.33 & & 4.67 & 4.00 \\
Cheap talk (CT) & 0.11 & 0.44 & 0.44 & 0.33 & & 4.67 & 4.00 \\
\hline
\end{tabular}

Table 2: Predicted frequencies of entry levels and payoffs in the (symmetric) equilibria in the various treatments.

On basis of the numbers in the table we can formulate the following two hypotheses:

H.1-1. The level of efficiency and the payoffs obtained in the CT treatment equals that of the NI treatment.

H.1-2. The level of efficiency and the payoffs obtained in the FI treatment exceeds that of the CT treatment and the NI treatment. 
One reason to expect the first hypothesis to be rejected is that in cheap talk experiments overcommunication is a rather robust and frequently recurrent phenomenon (though, it is not clear that this phenomenon is robust to our extension to multiple strategically interacting receivers). That is, senders' messages typically convey more (truthful) information than is predicted by sequential equilibrium prediction (assuming pure payoff-oriented and selfcentered players), which is picked up by the receivers. If this is found to be true in the present setting, then cheap talk has the potential to increase the level efficient entry and payoffs relative to the NI treatment. Our base hypotheses with respect to the information transmission and processing are formulated relative to the standard theoretical prediction (cf. Crawford and Sobel, 1982):

H.2-1. In the CT treatment, the frequency of chosen messages is constant over states.

H.2-2. In the CT treatment, the likelihood to enter is constant over messages.

The combination of these two hypotheses, provides us with a third testable hypothesis:

H.2-3. In the CT treatment, the likelihood to enter is constant over states.

Although it is to be expected that the latter hypothesis will only be rejected in case at least one of the former hypothesis is rejected, not being able to reject this hypothesis may go handin-hand with rejecting both of the former two hypotheses (but not with rejecting only one of them).

\subsection{Design and procedures}

For the two baseline treatments (NI and FI) we had in total five matching groups, while we ran the cheap talk treatment (CT) with eight matching groups. All matching groups consisted of twelve subjects, who played the game as specified by the treatment over a sequence of fifty rounds.

Every round again the subjects in a matching group were randomly partitioned into four triples and, for each triple, one member was assigned the sender role while the other two members got one of the receiver roles. Next, a state was drawn at random and the sender was informed on the selected state. The information the receivers received depended on the respective treatment. In the FI treatment, also the receivers were informed about the state. In the NI treatment, the receivers did not get any information. In the CT treatment, the only information receivers held was the message that was sent to them by the sender. To do so, the sender had three messages at her disposal that were of the type "the real state is

...". Given the information, next, the receivers had to choose whether to enter or to stay out. 
Depending on the choices of the receivers and the state drawn, the three members obtained their respective payoff.

The phrasing in the experiment was chosen as neutral as possible: the sender and receiver roles were named role $\mathrm{A}$ and role $\mathrm{B}$, the states where presented as payoff tables 1, 2 and 3, the messages were numbered similarly ("Table ... has been selected"), and the actions were called option $\mathrm{X}$ and option $\mathrm{Y}$. To facilitate learning, during the session, each subject had a history table displaying the table selected, her role, the decisions by the members in their triple, and her payoff. After the last round of play, three rounds were randomly drawn with replacement and subjects were paid according to the sum of the payoffs they made in the three rounds drawn. The rematching in triples within matching groups, the random role assignment and the lottery payment were implemented to mimic a one-shot interaction between subjects as much as possible.

Subjects were invited and could sign up to participate in an economic experiment via ORSEE (Greiner, 2004). The sessions were run in the BEElab at Maastricht University in May and June 2012. The instructions and comprehension questions were paper-based; the decision phase was computerized using z-Tree (Fischbacher, 2007). In total 216 students participated in our sessions. An experimental session lasted between 60 and 90 minutes and the average earning of the subjects was 13.41 Euro, including a 3 Euro show-up fee.

\section{Results}

In total we have five independent observations for the baseline treatments (NI and FI) and eight for the treatment with cheap talk (CT). We do not find any substantial time trends in

period averages for each matching group. ${ }^{1}$ Therefore, we consider on matching group averages over all periods in our data analysis.

\subsection{Treatment comparisons}

In this first subsection, we focus on the outcomes generated in the various treatments and the performance of the cheap talk treatment in particular. Thereby, we focus on the number of entrants in the various states and the average payoffs of the sender and the receiver(s).

Figure 1 presents the frequencies of the number of entrants in the different states for the three treatments. We see that in the FI treatment, there have been no instances where two receivers entered in the small state and where no receiver entered in the large state. From this we can conclude that the structure of the payoffs has been clear to all participants. Furthermore, we see that the differences between the NI treatment and the CT treatment

\footnotetext{
${ }^{1}$ All instructions, software, data files (raw and processed) and codes used for analysis are retrievable from the second authors personal webpage.
} 


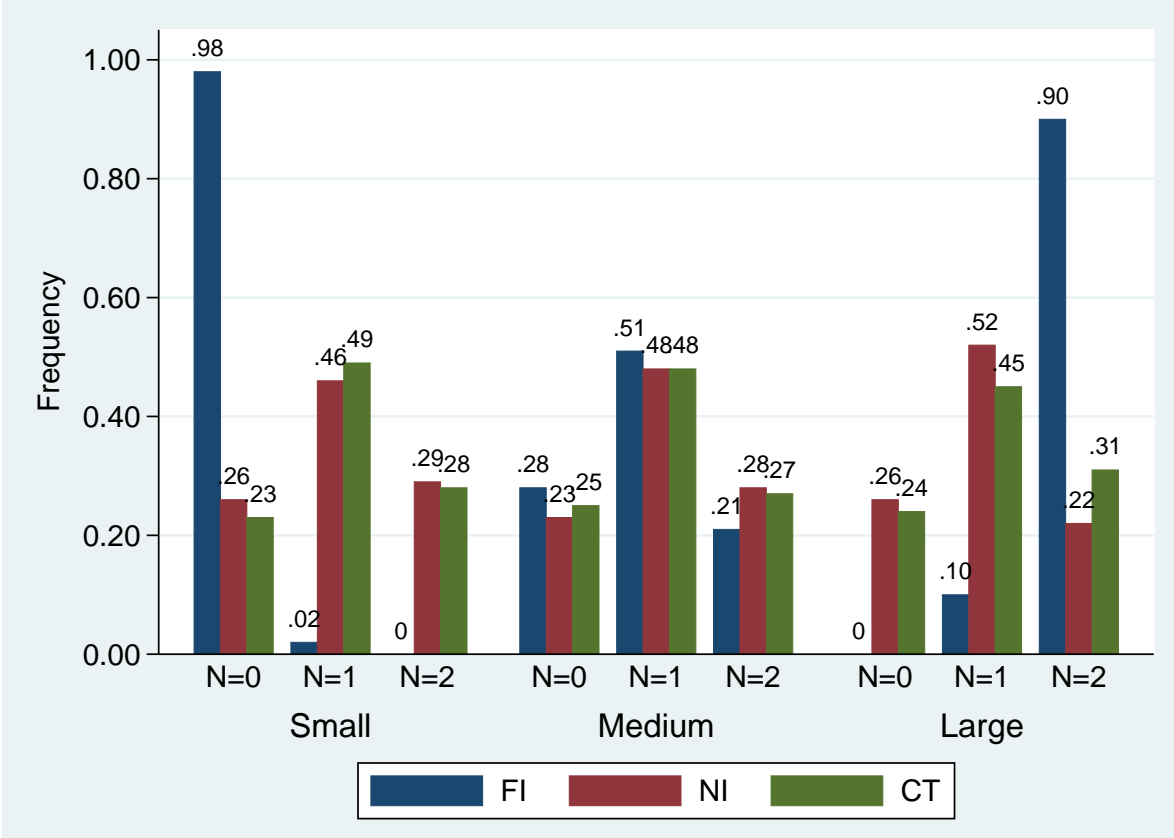

Figure 1: Frequency of the number of entrants.

are rather small. Only in the large state there is a small difference visible; here, the expected number of entrants is 0.96 in the NI treatment and 1.07 in the CT treatment. Where cheap talk communication appears to successfully support entry in the large state, it fails to prevent receivers from entering in the small state. Yet another striking observation is that the entry outcomes are quite similar across all treatments in the medium state. While this is close to symmetric equilibrium prediction for the FT treatment, for the NI treatment and the CT treatment there is substantially less entry compared to symmetric equilibrium prediction.

Next, we compare the performance of the three treatments on basis of two efficiency criteria. The first efficiency criterium relates to the frequency of efficient entry levels in the different states. In a monopolistic competitive market, firms usually enter up to the moment that all excess profits from entry are eroded. In our configuration, this would mean that (if all information were to be available) there should be no entry in the small state, one entrant in the medium state and two entrants in the large state. Table 3 shows for each treatment the frequency of efficient entry outcomes per state and on average over states.

The frequencies of efficient entry levels for the FI treatment are quite in line with the theoretical predictions in Table 2. For the other two treatments the frequency of efficient outcomes is larger in comparison to theoretical prediction when the state is small or medium, but lower in case the state is a large. These opposite effects lead to the overall frequency of efficient entry levels not being substantially different as predicted by theory. 


\begin{tabular}{lccccccc}
\hline & \multicolumn{3}{c}{ Frequency of efficient entry outcomes } & & \multicolumn{2}{c}{ Average payoff } \\
\cline { 2 - 4 } \cline { 8 - 9 } Treatment & Small & Medium & Large & Overall & & Sender & Receiver \\
\hline Full info (FI) & 0.98 & 0.51 & 0.90 & 0.80 & & 5.44 & 4.69 \\
No info (NI) & 0.26 & 0.48 & 0.22 & 0.32 & & 5.29 & 4.11 \\
Cheap talk (CT) & 0.23 & 0.48 & 0.31 & 0.34 & & 5.24 & 4.14 \\
\hline
\end{tabular}

Table 3: Frequency of efficient entry outcomes and payoffs in the various treatments.

On the overall level, the frequency of efficient entry outcomes in the CT treatment does not differ from that in the NI treatment (Mann-Whitney, two-sided: $p=.5549$ ). With regard to these frequencies on state-level, there are no significant differences between these two treatments in the small state $(p=.2403)$ and the medium state $(p=.2403)$. However, and as was already visible in Figure 1, cheap talk communication induces more efficient entry in the larger state $(p=.0475)$. But, this effect is not sufficient to make cheap talk communication effective on the overall level.

Comparing these two treatments with the FI treatment, there is no difference in the medium state (CT: $p=.4624$; NI: $p=.6004$ ). However, in the small state and large state, the frequency of efficient entry outcomes in the FI treatment significantly exceeds that of the NI and CT treatment (all tests: $p<.01$ ). These differences are sufficiently strong to make the FI treatment more efficient than the other treatments on the overall level (both test: $p<.01)$.

With respect to the expected payoffs, our findings are comparable to those for the frequencies: There is no significant difference between expected payoffs in the CT treatment and the NI treatment (sender: $p=.7140$; receivers: $p=.5582$ ) and the expected payoffs in the $\mathrm{CT}$ treatment are below that in the FI treatment (sender: $p=.0669$; receivers: $p=.0034$ ).

Result 1-1. Cheap talk communication fails to increase efficiency and payoffs beyond the level that is obtained in a setting where receivers do not receive any information on the actual state.

Result 1-2. The level of efficiency and payoff that is obtained in a setting where receivers are perfectly informed about the state cannot be achieved when receivers are informed via messages send by the sender.

In order to better understand why cheap talk communication failed to enhance efficiency relative to the situation where receivers do not obtain any information regarding the state in our experiments, we continue with an analysis of the senders' and receivers' behavior in the cheap talk treatment. 


\subsection{Individual behavior}

Table 4 shows the probabilities by which the senders have chosen messages in the different states. One property that stands out is that in each column the number on the principal

\begin{tabular}{lccc}
\hline & \multicolumn{3}{c}{ Message } \\
\cline { 2 - 4 } State & Small & Medium & Large \\
\hline Small & 0.69 & 0.22 & 0.10 \\
Medium & 0.50 & 0.41 & 0.10 \\
Large & 0.59 & 0.22 & 0.19 \\
\hline Overall & 0.59 & 0.28 & 0.13 \\
\hline
\end{tabular}

Table 4: Messages chosen by the senders in the different states.

diagonal exceeds the two numbers off the diagonal. Since the signaling behavior of the sender does not differ much across matching groups, it does not come as a surprise that we can reject the hypothesis that probabilities by which messages are chosen are independent of the state: Given a message, the probability by which this message is chosen is significantly higher in the state that matches the message compared to the other two states (Wilcoxon, two-sided, $p<.03$ for all six comparisons) and are not significantly different between the other two states ( $p>.12$ for all three comparisons).

Result 2-1. The messages send by the sender do convey information on the actual state.

The dominance of the diagonal in the matrix that reflects signaling behavior in the cheap talk treatment, indicates that on the aggregate level senders tell the truth excessively compared to standard theoretical prediction. So, the excessive truth-telling that as been found in earlier literature is robust to introducing multiple strategically interacting receivers. The numbers in the table also indicate a general tendency for strategic deception (understating the state) and a substantial amount of obscure deception (overstating the state). We will provide a further discussion on these two forms of deception in the concluding section.

In order to estimate the amount of truth-telling, we decompose the sender's signaling behavior into a truth-telling component and a state-independent component:

$$
\left(\begin{array}{ccc}
m_{S, S} & m_{S, M} & m_{S, L} \\
m_{M, S} & m_{M, M} & m_{M, L} \\
m_{L, S} & m_{L, M} & m_{L, L}
\end{array}\right)=\lambda\left(\begin{array}{ccc}
1 & 0 & 0 \\
0 & 1 & 0 \\
0 & 0 & 1
\end{array}\right)+(1-\lambda)\left(\begin{array}{ccc}
\sigma_{S} & \sigma_{M} & \sigma_{L} \\
\sigma_{S} & \sigma_{M} & \sigma_{L} \\
\sigma_{S} & \sigma_{M} & \sigma_{L}
\end{array}\right)
$$

and estimate the four parameters that fit our data best: ${ }^{2}$

$$
\lambda=0.1365 \quad \sigma_{S}=0.62 \quad \sigma_{M}=0.27 \quad \sigma_{L}=0.10 .
$$

\footnotetext{
${ }^{2}$ The parameters listed minimize the sum of the distances to the eight independent observations.
} 
These numbers indicate, first, that if we were to partition the population into truth-tellers and perfect babblers, we would estimate about $14 \%$ of the population to consist of truth-tellers, and, second, that the perfect babblers have a general tendency to understate the state.

Now we know that messages do convey information, the next question is whether this is picked up by the receivers. Table 5 presents the entry decisions of the receivers in response to messages received. We see that the probability by which the receivers enter are increasing

\begin{tabular}{lcc}
\hline & \multicolumn{2}{c}{ Entry decision } \\
\cline { 2 - 3 } Message & In & Out \\
\hline Small & 0.45 & 0.55 \\
Medium & 0.55 & 0.45 \\
Large & 0.81 & 0.19 \\
\hline Overall & 0.53 & 0.47 \\
\hline
\end{tabular}

Table 5: Entry decisions of the receiver after the different messages.

in the message, and find that the differences are significant (S-M: $p=.0251$; M-L: $p=$ .0117). This result implies that the excess truth-telling is picked up by the receivers and their responses reflect some trust in the messages.

Result 2-2. Receivers respond to messages in a way that reflects that they regard messages somewhat trustworthy.

Table 6 combines the information presented in the previous two tables and shows the probability that the receivers enter for each state. We see that the receivers' entry probabilities for

\begin{tabular}{lcc}
\hline \multirow{2}{*}{ State } & \multicolumn{2}{c}{ Entry decision } \\
\cline { 2 - 3 } Small & In & Out \\
\hline Medium & 0.51 & 0.49 \\
Large & 0.54 & 0.47 \\
\hline Overall & 0.53 & 0.46 \\
\hline
\end{tabular}

Table 6: Entry decisions of the receiver in the different states.

the three states are quite close. Yet, the probability by which receivers enter is significantly lower in the small state compared to the other two states and there is no significant difference between the larger two states (S-M: $p=.0294$; S-L: $p=.0173$; M-L: $p=.3991$ ).

Result 2-3. Cheap talk communication results in minor differences in entry decisions across states. 
In sum, we find that messages are informative and this has been picked up by the receivers, but that the receivers' entry decisions in response to the messages is such that it does not produce sufficient differences in entry probabilities across states for cheap talk to be able to enhance efficiency.

\section{Discussion}

In this final section we first aim to provide an explanation for why, despite senders' messages conveying information and the receivers recognizing this and responding to that, cheap talk does not induce an enhancement of efficiency levels and payoffs beyond that obtained without any information transmission. Second, we try to increase understanding in the senders' substantive use of messages that are destructive for the payoffs of all parties involved by investigating several alternative underlying motives that may play a role.

\subsection{Possible explanation}

Table 7 shows the entry probabilities for the receivers in the symmetric equilibrium, given the sender's behavior in the experiment. Comparing these numbers with those in Table 5, we see

\begin{tabular}{lcc}
\hline & \multicolumn{2}{c}{ Entry decision } \\
\cline { 2 - 3 } Message & In & Out \\
\hline Small & 0.61 & 0.39 \\
Medium & 0.64 & 0.36 \\
Large & 1.00 & 0.00 \\
\hline Overall & 0.67 & 0.33 \\
\hline
\end{tabular}

Table 7: Equilibrium behavior of the receivers given the sender's behavior.

that, while receivers do realize that the senders messages capture some amount of truthful information, they fail to respond optimally. Receivers simply behave overly cautious after every possible message. This cautiousness was something that was also observed in the NI treatment, where receivers only enter with $51 \%$ probability, while equilibrium predicts them to enter with $67 \%$ probability.

One obvious candidate explanation for the overly cautiousness to enter is risk aversion. In the post-experimental questionnaire we elicited our participants' risk attitudes by the direct approach as suggested in Dohmen et al. (2011). We find that participants in the NI treatment report a slightly higher degree of risk aversion compared to the FI treatment $(p=.0729) .^{3}$

\footnotetext{
${ }^{3}$ We asked the participants the question "How do you see yourself: Are you generally a person who is fully prepared to take risks or do you try to avoid taking risks?". Next they were asked to tick a box on a scale from 0 to 10 where the value 0 means: "not at all willing to take risks" and the value 10 means "very willing to take risks". The average response was $6.06,5.38$ and 5.96 in respectively the FI, NI and CT treatment.
} 
In the FI treatment the receivers enter with $47 \%$ probability in the medium state, which is close to the symmetric equilibrium prediction. So, risk attitude can explain (in part) the low entry rate in the NI treatment. However, participants in the CT treatment do not report a different risk attitude compared to those in the FI treatment $(p=.8103)$. Therefore, risk aversion is not the likely explanation for low entry rates in the CT treatment. Yet, apart from the uncertainty about the other receiver's behavior, in the CT treatment, there is also uncertainty regarding the actual state being drawn while this type of uncertainty is not present in the FI treatment. So, the difference may be explained by the difference in the nature of the risk across treatments. ${ }^{4}$

\subsection{Obscure deception}

Despite there being excess truth-telling on the aggregate level, only in $43 \%$ of the cases senders told the truth. Obviously, our setting invites senders to deceive the receivers by understating the true state and it is not too surprising to find this to happen. However, in about onefourth of the instances where a sender sent a deceptive message, she actually overstates the state - and this number is stable over time and across matching groups. Where there are clear strategic motives for understating the state (strategic deception), these overstatements appear somehow a bit obscure (obscure deception). Still, about three-fourth of the subjects at least once sent such an obscure deceptive message and about one-fourth of the subjects sent such a message in at least $20 \%$ of the instances they acted as sender. Given the large number of individuals using such a message and the use not vanishing over time, we further investigate on possible motives for using such a message.

Table 8 shows the costs and gains from lying for the sender and receiver, given the actual response to the messages by the receivers in the experiment. The first number in each cell corresponds to the gain to the sender of sending the respective message in the respective state; the second number corresponds to the gain for the receivers. We see that it is always

\begin{tabular}{lccc}
\hline & \multicolumn{3}{c}{ Message } \\
\cline { 2 - 4 } State & Small & Medium & Large \\
\hline Small & - & $-0.42,-0.32$ & $-1.44,-1.26$ \\
Medium & $+0.42,+0.11$ & - & $-1.01,-0.44$ \\
Large & $+1.44,-0.53$ & $+1.01,-0.32$ & - \\
\hline
\end{tabular}

Table 8: Costs and gains from lying.

\footnotetext{
${ }^{4}$ Actually, in case receivers have in mind to behave in the NI treatment as they would do in the FI treatment and take the average over these probabilities (because all states are equally likely to be drawn), this can explain the close to $50 \%$ entry. If they would indeed compound the probabilities in such a way, they falsely ignore the payoff differences across states (note here that the payoff differences between the small and medium state equal 2, while the difference between the medium and large state equals 3 ).
} 
beneficial for the sender to understate the actual state. Where these lies are harmful for the receivers in the large state, they are beneficial for them in the medium state. The reason for this is that the receivers enter with $55 \%$ probability (recall Table 5) after receiving the message saying that the state is medium, which is too high in the medium state. Sending the message saying that the state is small in this state helps the receivers in suppressing the entry probability to $45 \%$, which happens to be beneficial for the receivers.

Overstating the actual state is always harmful for the sender and the receivers. It is questionable why in $32 \%$ of the instances in the small state and in $10 \%$ of the instances in the medium state such messages have been chosen by the senders. A strategic motive may be to spoil the communication channel in order to implement a babbling equilibrium. However, since the interaction properties in our experimental mimic that of a one-shot interaction (coplayer rematching, re-assignment of roles, anonymous decision making, not all rounds are selected for payment, and in addition there is variation in states being selected), it is hard to built a babbler reputation. Therefore, this strategic motive seems not to be a plausible explanation for the high amount of obscure deceptions.

One other experimental study in which nonstrategic deception has been found is ClotsFigueras et al. (2012). That study considers a version of the trust game where the investor is not aware of the possible benefit on investment. The investee, who is aware of this benefit, has the capacity to transmit information on it to the investor prior to his investment decision. The investee has a clear strategic motive to overstate the benefit in order to persuade the investor to invest a lot. Although more instances are found in which investees overstate the benefit, there are a substantial amount of instances in which they understate the true benefit. Their explanation for these nonstrategic deceptions is guilt aversion: by understating the benefit, investees decrease the return expectations of the investors, thereby decreasing their guilt from a possibly disappointing return. As in our situation the sender of the message has no further actions to take, guilt considerations seem not to play any role.

Another emotional motive for sending such a lie, that is given by Gneezy (2005), is a "spiteful reaction to unfair behavior". Therefore these lies are classified "spiteful black lies" in Erat and Gneezy (2012). ${ }^{5}$ As participants in our experiment have been rematched and reassigned their role every round anew, such a spiteful reaction typically targets someone that cannot be held accountable for having caused a feeling of being treated unfairly. Nevertheless the spiteful reaction may be a mere act of getting rid of such negative feelings. Two prominent experiences that we recognize as potential triggers for spiteful response are: (1) having suffered from a lie as a receiver, and (2) having suffered from not having been trusted as a sender

\footnotetext{
${ }^{5}$ Note that what we call obscure deceptions is close to what Erat an Gneezy call spiteful black lies, but not synonym. The reason is that the negative consequences of these lies for are players involved is according to payoff expectations based on the experimental data.
} 
after having told the truth. Out of the 202 instances of obscure deceptions from the second round onwards, only (1) thirteen $(6.44 \%)$ were sent after having received a payoff lower than 4 after a lie has been told to them as a receiver in the previous round, and (2) only eighteen $(8.91 \%)$ were sent after having received a payoff below 5 after having told the truth in the small or medium state as a sender in the previous round. From these numbers we infer that the high amount of obscure deceptions is not likely to be explained by spiteful reactions to unfair behavior.

\section{References}

1. Battaglini M and U Makarov (2012). Cheap talk with multiple audiences: An experimental analysis. Working paper.

2. Cai H and J Wang (2006). Overcommunication in strategic information transmission games. Games and Economic Behavior 56(1): 7-36.

3. Clots-Figueras I, R Hernán González and P Kujal (2012). Trust me I am (not) lying: Message inflation and deflation in the investment game. Working paper.

4. Crawford V and J Sobel (1982). Strategic information transmission. Econometrica 50(6): 1431-1451.

5. Dickhaut J, K McCabe and A Mukherji (1995). An experimental study on strategic information transmission. Economic Theory 6(3): 389-403.

6. Dohmen T, A Falk, D Huffman, U Sunde, J Schupp and G Wagner (2011). Individual risk attitudes: Measurement, determinants and behavioral consequences. Journal of the European Economic Association 9(3): 522-550.

7. Erat S and U Gneezy (2012). White lies. Management Science 58(4): 723-733.

8. Farrell J and R Gibbons (1989). Cheap talk with two audiences. The American Economic Review 79(5): 1214-1223.

9. Fischbacher U (2007). zTree: Zurich toolbox for ready-made economic experiments. Experimental Economics 10(2): 171-178.

10. Gneezy U (2005). Deception: The role of consequences. The American Economic Review 95(1): 384-394. 
11. Greiner B (2004). An online recruitment system for economic experiments. In: K Kremer and V Macho (eds.): Forschung und wissenschaftliches Rechnen 2003. GWDG Bericht 63, Göttingen: Ges. für Wiss. Datenverarbeitung, pp. 79-93.

12. Sánchez-Pagés S and M Vorsatz (2007). An experimental study of truth-telling in a sender-receiver game. Games and Economic Behavior 61(1): 86-112.

13. Sobel J (2009). Signaling games. In: R Meyers (ed.) Encyclopedia of Complexity and System Science: pp. 8125-8139.

14. Wang J, M Spezio and C Camerer (2010). Pinocchio's pupil: Using eyetracking and pupil dilation to understand truth-telling and deception in sender-receiver games. The American Economic Review 100(3): 984-1007. 